\title{
COMPUTATION OF ENGINE NOISE PROPAGATION AND SCATTERING OFF AN AIRCRAFT
}

\author{
J. Xu*, D. Stanescu*, M.Y. Hussaini*, and F. Farassat ${ }^{\dagger}$ \\ *School of Computational Science and Information Technology, Florida State University \\ Tallahassee, Florida 32306-4120 \\ † NASA Langley Research Center, Hampton, Virginia 23681-0001
}

\begin{abstract}
The paper presents a comparison of experimental noise data measured in flight on a two-engine business jet aircraft with Kulite microphones placed on the suction surface of the wing with computational results. Both a time-domain discontinuous Galerkin spectral method and a frequency-domain spectral element method are used to simulate the radiation of the dominant spinning mode from the engine and its reflection and scattering by the fuselage and the wing. Both methods are implemented in computer codes that use the distributed memory model to make use of large parallel architectures. The results show that trends of the noise field are well predicted by both methods.
\end{abstract}

\section{INTRODUCTION}

The fan inlet and exhaust noise represents one of the major components of the noise signature of an aircraft at take-off and landing. The noise radiated to the far field by the engine of an aircraft is largely influenced both by the flow around the wing and fuselage and by the scattering from various other aircraft surfaces. In principle, it is possible to reduce the aircraft noise footprint by taking advantage of engine and wing location and manipulating the flow around the aircraft. Experimental investigations of these phenomena are difficult to perform and extremely expensive. Numerical simulations offer a relatively inexpensive alternative, and such simulations are becoming increasingly attractive due to the recent advances in both computer architecture and computational methods. To date, most measurements and modeling of engine noise are confined to isolated engines $[1,2,3]$. However, Stanescu, Hussaini, and Farassat [4] have recently computed the engine noise propagation and scattering for a generic aircraft configuration by numerically solving the Euler equations by a discontinuous Galerkin spectral element method. The recent popularity of such methods in aerodynamic applications stems from the fact that they require relatively fewer points per wavelength, they have low dispersion and dissipation errors $[5,6,7]$, they have geometric flexibility, and they are compact, robust and inherently parallelizable $[4,8]$. In a more recent work $[9]$, the same authors presented a simultaneous research initiative consisting in the development of a spectral element method for the solution, in the frequency domain, of the acoustic potential equation in the presence of a non-uniform flow field. The two methods are believed to be complementary tools useful for prediction of the tonal sound field in the near field of the engine.

The purpose of the present effort is to investigate the feasibility of large-scale aircraft noise simulations, and validate them with the available experimental data. To that end, we employ the aforementioned numerical methodology in the investigation of engine noise propagation and scattering off an actual two-engine jet aircraft. After a brief discussion of the two methods in the next section, we present the nu-

Copyright (C)2003 by D. Stanescu. Published by the American Institute of Aeronautics and Astronautics, Inc. with permission. 
merical results obtained for several flight conditions and compare them with the available experimental data. Although the modal composition of the source could not be obtained experimentally and the actual flight conditions could not be replicated exactly in the computations presented here, results show that the noise signature obtained from computations matches the trend of the experimental data.

\section{PROBLEM FORMULATION AND SOLUTION TECHNIQUE}

\section{Computational model}

We assume that the engine noise source is known and consider noise propagation and scattering in the left half space implicitly assuming symmetry of the problem about the $y=0$ plane which is chosen to bisect the aircraft along the fuselage. For the computational purpose, the half space is truncated into a computational domain comprised of a bounded physical domain with a damping layer surrounding it. The latter is used to ensure the physical domain remains uncontaminated by reflections. The surface that separates the computational domain from the surrounding medium is denoted by $\Gamma_{\infty}$. This governing equations are solved in nondimensional form. The reference quantities for non-dimensionalization are: $\rho_{\infty}$ for the density, $c_{\infty}$ for the velocity components, $\rho_{\infty} c_{\infty}^{2}$ for the pressure, the radius $R$ of the noise-source disk for distance, and $\frac{R}{c_{\infty}}$ for time. The total domain of computation (including the damping layers) is defined in the non-dimensional Cartesian coordinates as $21.4 \leq x \leq 40.0,-12.0 \leq y \leq 0.0$ and $-1.8 \leq z \leq 11.2$. The computational domain with the embedded aircraft is depicted in Fig. 2. As the propagation distance is relatively small, viscous effects are neglected and the problem is assumed to be governed by inviscid compressible flow equations.

The computational domain is covered by a grid of non-overlapping general hexahedral elements that can have curved boundaries. The ICEMCFD Hexa commercial package is used to generate the unstructured hexahedral grid around the aircraft configuration. Once an unstructured grid of hexahedra is generated, an attractive new feature of this package allows for the generation of points along each of the edges of the hexahedral mesh, which can be either a Legendre-Lobatto or a Chebyshev-Lobatto distribution for a specified polynomial of degree $N$. Fig. 3 shows the hexahedral representation of the underlying geometry with Gauss-Legendre point distribution, where $N=5$. All the necessary point coordinates can then be computed by interpolation based on the spectral interpolants along the edges (to obtain coordinates at the Gauss points from the Lobatto points on the edges) followed by three-dimensional transfinite interpolation [10] on the faces and inside the elements.

\section{Boundary conditions}

A zero normal velocity boundary condition is imposed on the symmetry surface $y=0$ (this supposes that the engines are symmetrically placed on either side of the fuselage and rotate in opposite sense) as well as on the fuselage, nacelle and wing surfaces. The boundary conditions on the other sides of the computational domain that make up $\Gamma_{\infty}$ are treated by a damping layer method [11]. The damping layer is about 3.5 wide in the $x-, y-$ and $z$-directions. Waves incident on this layer are damped and reflections into the physical domain of interest are minimized. This is obtained by modifying the governing equations through the addition of a damping term in the form

$$
\frac{\partial Q}{\partial t}+\nabla \cdot F=-\sigma(\mathbf{x}) Q
$$

where the damping parameter is made to vary from 0 at the interior limit of the damping layer to a maximum value on $\Gamma_{\infty}$ according to a power law

$$
\sigma(\mathbf{x})=\sigma_{M} \sum_{i}\left(\frac{x_{i}-x_{i}^{i n t}}{x_{i}^{e x t}-x_{i}^{i n t}}\right)^{\beta}
$$

with $x_{i}^{i n t}$ and $x_{i}^{e x t}$ the coordinates of the interior and exterior limits of the absorbing layer, limits that lie along planes on which one coordinate is 
constant.

The engine tone noise source is specified as a combination of spinning modes on a surface $\Gamma_{f}$ conveniently situated inside the nacelle. For a single spinning mode with azimuthal order $s$ and radial order $d$, usually denoted as $(s, d)$, the perturbation of the flow variables from the mean flow quantities (denoted by bars) is given by $[11,12]$ :

$$
\left(\begin{array}{c}
p-\bar{p} \\
\rho-\bar{\rho} \\
v_{x}-\bar{v}_{x} \\
v_{r}-\bar{v}_{r} \\
v_{\theta}-\bar{v}_{\theta}
\end{array}\right)=A\left(\begin{array}{c}
E_{m}\left(k_{m d} r\right) \cos \Theta \\
\frac{1}{\bar{c}^{2}} \cdot E_{m}\left(k_{m d} r\right) \cos \Theta \\
\frac{k_{x}}{\omega_{r} \overline{\bar{\rho}}} \cdot E_{m}\left(k_{m d} r\right) \cos \Theta \\
\frac{\underline{r}_{x d}}{\omega_{r} \bar{p}} \cdot E_{m}^{\prime}\left(k_{m d} r\right) \sin \Theta \\
\frac{m}{r \omega_{r}} \cdot E_{m}\left(k_{s d} r\right) \cos \Theta
\end{array}\right),
$$

where $\Theta=k_{x} x+m \theta-\omega_{r} t, \quad k_{m d}=$ $\sqrt{\left(\omega_{r} / c\right)^{2}-{k_{x}}^{2}}$, and $\omega_{r}=\frac{\omega \cdot R}{c_{\infty}}$. The function $E_{m}\left(k_{m d} r\right)=J_{m}\left(k_{m d} r\right)+q Y_{m}\left(k_{m d} r\right)$ is the duct eigenfunction with $J_{m}$ and $Y_{m}$ denoting the Bessel functions of the first and second kind, respectively. The noise source is specified on the circular disk centered at $(34.7,4.6,5.3)$.

\section{Time domain formulation}

For the time domain formulation, the governing equations are considered the Euler equations in Cartesian conservation form,

$$
\frac{\partial \tilde{Q}}{\partial t}+\sum_{d=1}^{3} \frac{\partial \tilde{F}_{d}}{\partial x_{d}}=0 .
$$

where the state vector $\tilde{Q}$ and the flux vector $\tilde{F}_{d}$ are given by

$$
\tilde{Q}=\left(\begin{array}{c}
\rho \\
\rho v_{1} \\
\rho v_{2} \\
\rho v_{3} \\
\rho E
\end{array}\right), \tilde{F}_{d}=\left(\begin{array}{c}
\rho v_{d} \\
\rho v_{1} v_{d}+p \delta_{1 d} \\
\rho v_{2} v_{d}+p \delta_{2 d} \\
\rho v_{3} v_{d}+p \delta_{3 d} \\
(\rho E+p) v_{d}
\end{array}\right),
$$

with $\rho$ the fluid density, $E$ the internal energy, $p$ the pressure, and $v_{d}(d=1,2,3)$ the velocity components.

Each element of the grid is mapped onto the master element $\Omega_{M}=[-1,1]^{3}$ with an isoparametric transformation for the expediency of representing the solution in each element by spectral basis functions defined on the interval $[-1,1]$. Under the mapping, Eq. (4) becomes

$$
\frac{\partial Q}{\partial t}+\sum_{d=1}^{3} \frac{\partial F_{d}}{\partial \xi_{d}}=0
$$

where $Q$ and $F$ are the transformed components of the state and flux vectors

$$
Q=J \tilde{Q}, \quad F_{d}=J \sum_{m=1}^{3} \frac{\partial \xi_{d}}{\partial x_{m}} \tilde{F}_{m},
$$

and $J$ is the Jacobian of the transformation. The computational space coordinates are denoted by either $\left(\xi_{1}, \xi_{2}, \xi_{3}\right)$ or $(\xi, \eta, \zeta)$ hereafter for convenience.

Let the space of polynomials of degree $N$ in $\xi \in[-1,1]$ be denoted by $P_{N}$. A basis for this space can be constructed using the Lagrange interpolating polynomials $h_{j}, j=0,1, \ldots, N$, through the $N+1$ Gauss-Legendre [13] quadrature nodes $\xi_{i}, i=0,1, \ldots, N$. A discontinuous Galerkin approximation is obtained by requiring

$$
\left(Q_{t}, \phi_{i j k}\right)+\left(\nabla_{\xi} \cdot F, \phi_{i j k}\right)=0
$$

where $(\cdot, \cdot)$ represents the usual $L^{2}$ inner product, and $\phi_{i j k}=h_{i}(\xi) h_{j}(\eta) h_{k}(\zeta)$ are the basis functions of $P_{N}^{3}$.

Using the divergence theorem and Gauss quadrature, expanding the boundary integral and performing some algebraic manipulation, the final discrete form of the equations governing each variable at the Legendre-Gauss points are given by

$$
\frac{d Q_{i j k}}{d t}=-\left[D^{\xi}+D^{\eta}+D^{\zeta}\right] F,
$$

where the right-hand side is a sum of discrete differential operators acting on the flux values of an element, which include values on the element faces. Here, the differential operator $D^{\xi} F$, for example, is defined as

$$
\begin{gathered}
D^{\xi} F=\frac{1}{w_{i}}\left[F_{1}^{*}\left(1, \eta_{j}, \zeta_{k}\right) h_{i}(1)-\right. \\
\left.F_{1}^{*}\left(-1, \eta_{j}, \zeta_{k}\right) h_{i}(-1)-d^{\xi} F\right],
\end{gathered}
$$


where $F^{*}$ denotes a common face flux, which can be computed directly from the readily available values of the state vector. $D^{\eta} F$ and $D^{\zeta} F$ follow by obvious permutations.

As the solution is approximated by a polynomial that passes through interpolation nodes distributed within the elements, a mismatch ensues when the interpolants are evaluated at element interfaces. This mismatch in the solution at element boundaries is resolved by solving the Riemann problem for the flux there (just as in the finite volume method) $[14,15]$. This leads to a semidiscrete form of Euler equations, which is simply an ordinary differential equation (ODE) system. The resulting ODE system is integrated in time using a low-storage Runge-Kutta scheme optimized for wave propagation [16]. Acoustic perturbations are obtained at each time step by subtracting the mean flow from the total flow variables, and the RMS pressure is obtained by integrating in time the acoustic pressure. This integration is only performed after sufficient time is allowed for the acoustic signal from the source to propagate through the computational domain and establish a periodic acoustic field.

\section{Frequency domain formulation}

The equation governing the acoustic field is in this case obtained by considering the flow irrotational, so that the continuity equation becomes

$$
\frac{\partial \rho}{\partial t}+\nabla \cdot(\rho \nabla \Phi)=0
$$

where $\rho$ is the fluid density, and $\Phi$ is the total velocity potential, related to the velocity by $\mathbf{V}=\nabla \Phi$. Under the isentropic assumption, the momentum equation is reduced to an algebraic relation relating the density to the velocity potential as

$\rho=\left[1-(\gamma-1)\left(\frac{\partial \Phi}{\partial t}+\frac{(\nabla \Phi)^{2}-M_{\infty}^{2}}{2}\right)\right]^{\frac{1}{\gamma-1}}$

where $M_{\infty}$ is the far field Mach number and $\gamma$ the specific heats ratio. Consider the un- steady flow field resulting from the superposition of small acoustic perturbations, denoted by a prime, on a steady mean flow denoted by an overbar: $\rho=\bar{\rho}+\rho^{\prime}$ and $\Phi=\bar{\Phi}+\Phi^{\prime}$. The partial differential equation governing the acoustic perturbations is

$$
\frac{\partial \rho^{\prime}}{\partial t}+\nabla \cdot\left(\bar{\rho} \nabla \Phi^{\prime}+\rho^{\prime} \nabla \bar{\Phi}\right)=0
$$

with the following relation relating the acoustic density to the acoustic velocity potential, obtained by linearization of equation (12):

$$
\rho^{\prime}=-\frac{\bar{\rho}}{\bar{c}^{2}}\left[\frac{\partial \Phi^{\prime}}{\partial t}+\nabla \bar{\Phi} \cdot \nabla \Phi^{\prime}\right] .
$$

For a frequency domain approach, the acoustic potential is considered to be of the form $\Phi^{\prime}=$ $\phi(x, y, z) \exp (i \omega t)$. In view of a weak formulation, the governing equation (13) is multiplied by a test function $\Psi^{\prime}=\psi(x, y, z) \exp (-i \omega t)$ and integrated using the divergence theorem to yield

$$
\begin{aligned}
& \int_{\Omega} \psi \frac{\partial \rho^{\prime}}{\partial t} r d \Omega-\int_{\Omega} \nabla \psi \cdot\left(\bar{\rho} \nabla \phi+\rho^{\prime} \nabla \bar{\phi}\right) r d \Omega+ \\
& \int_{\Gamma_{f}} \psi\left(\bar{\rho} \nabla \phi+\rho^{\prime} \nabla \bar{\phi}\right) \cdot \mathbf{n} r d s=0
\end{aligned}
$$

On the aircraft surface both the mean flow and the acoustic normal velocity component are supposed to be zero, so the surface integrand cancels there. Furthermore, the integrand is set to zero artificially on the $\Gamma_{\infty}$ surface, which does not sensibly affect the computed solution since the acoustic field is strongly damped in the absorbing layer.

Let $\mathbf{Z}$ denote the complex vector space of functions that are continuous on $\Omega$, whose restrictions to an element are polynomials of degree at most $N$ in each variable, where $N$ is a specified integer, and $\mathbf{Z}_{\Gamma_{f}} \subset \mathbf{Z}$ the subset of functions that satisfy the Dirichlet boundary condition on the source surface $\Gamma_{f}$. Substituting the expression of the density from the linearized momentum equation, the following variational 
problem is obtained: find $\phi \in \mathbf{Z}_{D}$ such that

$$
\begin{aligned}
& \int_{\Omega} \frac{\bar{\rho}}{\bar{c}^{2}}\left[\omega^{2} \phi \psi+i \omega \bar{u}\left(\phi \psi_{x}-\psi \phi_{x}\right)+\right. \\
& i \omega \bar{v}\left(\phi \psi_{y}-\psi \phi_{y}\right)+i \omega \bar{w}\left(\phi \psi_{z}-\psi \phi_{z}\right)+ \\
& \left(\bar{u}^{2}-\bar{c}^{2}\right) \phi_{x} \psi_{x}+\left(\bar{v}^{2}-\bar{c}^{2}\right) \phi_{y} \psi_{y}+ \\
& \left(\bar{w}^{2}-\bar{c}^{2}\right) \phi_{z} \psi_{z}+\bar{u} \bar{v}\left(\phi_{x} \psi_{y}+\phi_{y} \psi_{x}\right)+ \\
& \bar{u} \bar{w}\left(\phi_{x} \psi_{z}+\phi_{z} \psi_{x}\right)+\bar{v} \bar{w}\left(\phi_{y} \psi_{z}+\phi_{z} \psi_{y}\right)- \\
& \left.\psi \sigma\left(i \omega \phi+\bar{u} \phi_{x}+\bar{v} \phi_{y}+\bar{w} \phi_{z}\right)\right] d \Omega=0
\end{aligned}
$$

holds for any $\psi \in \mathbf{Z}_{\Gamma_{f}}$.

The previous equation is discretized by a Chebyshev spectral element method. To this end, a basis for $\mathbf{Z}$ is constructed using tensor products of the Lagrange interpolants through the Chebyshev-Gauss-Lobatto points in the element. Upon evaluating the integrals, a complex linear algebra problem of the form $A\{\phi\}=\{b\}$ is obtained, where $\{\phi\}$ is the vector of point values of the complex-valued acoustic potential $\phi$. The solution of this system is obtained using a Schur complement domain decomposition method implemented using the Message Passing Interface (MPI) standard. The matrix is stored in sparse mode (i.e. only the non-zeroes are stored), with each processor only storing a number of lines in the matrix. Let us denote by $P$ the total number of processors, the computational domain $\Omega$ is subdivided in as many partitions, and the unknowns situated on the surface $\mathcal{B}$ which separates the partitions are numbered last in the system. For every processor $p$, there will be a number of unknown $\phi$ values located on $\mathcal{B}$. The vector of unknowns is partitioned as

$$
\{\phi\}=\left\{\phi_{I}^{1} \ldots \phi_{I}^{P} \phi_{B}\right\}
$$

where $\phi_{I}^{p}$ denotes all the unknowns in subpartition $p$ not located on $\mathcal{B}$. The right-hand side vector $\{b\}$ is partitioned accordingly. The ma- trix $A$ can then be written in the form

$$
A=\left(\begin{array}{cccc}
A_{I I}^{1} & 0 & \ldots & A_{I B}^{1} \\
0 & A_{I I}^{2} & \ldots & A_{I B}^{2} \\
\dot{1} & \dot{1} & \cdot & \cdot \\
A_{B I} & A_{B I}^{2} & \ldots & A_{B B}
\end{array}\right)
$$

and straightforward elimination of the terms below the main diagonal leads to

$$
\left(\begin{array}{cccc}
A_{I I}^{1} & 0 & \ldots & A_{I B}^{1} \\
0 & A_{I I}^{2} & \ldots & A_{I B}^{2} \\
\cdot & \cdot & \cdot & \cdot \\
0 & 0 & \ldots & S
\end{array}\right)\left\{\begin{array}{c}
\phi_{I}^{1} \\
\phi_{I}^{2} \\
\cdot \\
\phi_{B}
\end{array}\right\}=\left\{\begin{array}{c}
b_{I}^{1} \\
b_{I}^{2} \\
\cdot \\
b_{S}
\end{array}\right\}
$$

where $b_{S}=b_{B}-\sum_{p} A_{B I}^{p}\left(A_{I I}^{p}\right)^{-1} b_{I}^{p}$. The problem has thus been reduced to solving a reduced system with matrix $S=A_{B B}-\sum_{p} A_{B I}^{p}\left(A_{I I}^{p}\right)^{-1} A_{I B}^{p}$ for the points on $\mathcal{B}$ only, followed by a solution on each domain of the interior problem. The matrix $S$ is much denser than the original matrix $A$ and its direct computation and storage is not efficient or even possible. However, for an iterative method, only the action of $S$ on a vector is needed, and once the sparse, distributed, matrix $A_{B B}$ is formed, this action can be computed by matrix-vector multiplications and solutions with $A_{I I}^{p}$ which are local operations on processor $p$ and do not require communications, followed by accumulation in the global vector $\phi_{B}$. All computations can be conveniently implemented by use of the high level primitives in the PETSc [17] package for efficient solution of partial differential equations.

\section{RESULTS AND DISCUSSION}

\section{Experimental data}

The data available for comparison was collected in $M_{\infty}=0.3$ flight at 500 foot above ground level. The average Mach number at the source disk based on the mass flux through the engine is approximately $M_{f}=0.53$. The Blade Passing Frequency (BPF) tone was measured using several Kulite microphones located on the wing suction side at different angles from the 
nacelle axis as shown in Fig. 1, at a relatively high power setting of the engine. Modal composition of the signal could not be satisfactorily established, however there were indications that the dominating mode is the spinning mode with azimuthal order $m=22$, and $m=18$ is also expected to have a large contribution. The data is presented as Sound Pressure Level (SPL) values, normalized such that the value at 20 degrees corresponds to zero SPL.

\section{Time domain results}

Propagation of both modes was simulated in the time domain with a quiescent medium surrounding the aircraft in a first attempt to determine the dominating mode and conduct a first test of the method on this geometry. The BPF tone corresponds to a reduced frequency $\omega_{r}=26.3$. Propagation and radiation of modes $(18,0)$ and $(22,0)$ was computed separately using an unstructured grid with 103, 105 elements. The solution is approximated by a sixth-order Legendre polynomial in each element, raising the number of Gauss-Legendre discretization points to $22,270,680$ in the computational domain that includes the damping layers. The computations used one node (32 processors running at $1.1 \mathrm{GHz}$ ) of an IBM Regatta-type SP4 machine and each one lasted about 10 days. An arbitrary value has been used for the amplitude of the incoming mode, which is not known from the experiments. Therefore, for the purpose of data comparison, computational data was matched with experimental data at the 60 degree microphone location, where the peak in SPL was noticed experimentally.

Fig. 4 shows a snapshot of the acoustic pressure contours on the surface of the aircraft at non-dimensional time $t=44$, immediately before starting integration for the RMS pressure computation, for spinning mode $(18,0)$. The computation indicates that radiation of mode $(22,0)$ has a pattern that is completely different from the experimental data, presented in Fig. 5. However, mode $(18,0)$ seems to produce a SPL distribution on the wing with the same characteristics as the experiments, Fig. 6 .
The quantitative difference in levels may stem from the mean flow effect. Indeed, increasing the mean flow Mach number in the duct from its zero value used for the present results will determine the mode to be more cut-on, with an immediate effect that the main radiation lobe will hit the wing at a lower angle location. Thus, SPL levels at angles lower than 60 degrees are expected to increase, while decreasing at 70 degrees. The effect of the mean flow, on the other hand, is expected to be in the opposite sense, but not as strong. It must also be considered that other sources of noise, not modeled in this computation (i.e. airframe noise) will increase the value of the measured SPL data, and this effect will become more important at larger distances from the engine (lower angles in the figures).

\section{Frequency domain results}

The effect of the mean flow has not yet been attested with the time domain formulation, as our efforts concentrated lately on the development of the frequency domain code. The latter incorporates a solver for the mean flow around the given configuration (at this time based on an incompressibility assumption to avoid nonlinear iteration). The frequency domain code however has far larger memory requirements. To date, our largest computation with this code was done on the same mesh with 103,105 elements but with quartic elements. There are thus a total of $6,746,736$ discretization points in the mesh, which would generate a complex matrix with a total of 1.4 billion non-zeroes whose only storage in sparse mode necessitates approximately 20GB memory. This resolution does not allow us to handle the exact experimental conditions, in particular at larger distances from the nacelle where the mesh becomes coarser and the necessary number of points per wavelength is not reached. Therefore, we considered a test case in which the mean flow around the aircraft was modeled based on a far field Mach number $M_{\infty}=0.1$ and a fan face Mach number $M_{f}=0.2$. The problem was run on 192 processors of the same IBM machine, and conver- 
gence more than three orders of magnitude was achieved after 2.5 days. This clearly demonstrates the need for a better multi-level preconditioner for the reduced matrix $S$. We must point out, however, that to our knowledge this is the largest problem of this type reported in the literature. Moreover, solving the complex linear algebra problem associated with discretization of Helmholtz-type equations is a well-known issue in the numerical analysis and parallel computing community and no satisfactory solution has, to our knowledge, been found as of now. Results obtained from this simulation for mode $(18,0)$ are presented in Fig. 7 , which shows that the presence of the mean flow has the anticipated effects as discussed above.

\section{ACKNOWLEDGEMENTS}

The authors want to thank D. Weir and T. Wolownik for their help with the experimental data. The support of D. Stanescu by NASA grant NAG-1-01031 is gratefully acknowledged.

\section{References}

[1] A.V. Parrett, and W. Eversman, "Wave Envelope and Finite Element Approximations for Turbofan Noise Radiation in Flight," AIAA Journal, Vol. 24, No. 5, 1986, pp.753-760.

[2] M. Nallasamy, "Computation of Noise Radiation from Fan Inlet and Aft Ducts," Journal of Aircraft, Vol. 34, No. 3, 1997, pp.387-393.

[3] Y. Ozyoruk and L. N. Long, "Computation of Sound Radiating from Engine Inlets," AIAA Journal, Vol. 34, 1996, No. 5, pp.894-901.

[4] D. Stanescu, M.Y. Hussaini and F. Farassat, "Aircraft Engine Noise Scattering A Discontinuous Spectral Element Approach," AIAA Paper 2002-0800, Reno, NV.
[5] F.Q. Hu, M.Y. Hussaini and P. Rasetarinera, "An Analysis of the Discontinuous Galerkin Method for Wave Propagation Problems." Journal of Computational Physics Vol. 151, 1999, pp.921-946.

[6] D. Stanescu, D.A. Kopriva and M.Y. Hussaini. "Dispersion Analysis for Discontinuous Spectral Element Methods." Journal of Scientific Computing Vol. 15, 2000, pp.149171.

[7] P. Rasetarinera, M.Y. Hussaini and F.Q. $\mathrm{Hu}$, in Discontinuous Galerkin Methods, Lecture Notes in Computational Science and Engineering 11 407-412. Berlin: Springer-Verlag, 2000.

[8] S. Rebay, "Efficient Unstructured Mesh Generation by Means of Delaunay Triangulation and Bowyer-Watson Algorithm." Journal of Computational Physics Vol. 106, 1993, pp.125-138.

[9] D. Stanescu, M.Y. Hussaini and F. Farassat, "Large Scale Frequency Domain Numerical Simulation of Aircraft Engine Tone Noise Radiation and Scattering", AIAA Paper 2002-2586, 8th AIAA/CEAS Aeroacoustics Conference, Breckenridge, CO.

[10] W.J. Gordon and C.A. Hall, "Construction of curvilinear co-ordinate systems and applications to mesh generation." International Journal for Numerical Methods in Engineering Vol. 7, 1973, pp.461-477.

[11] D. Stanescu, D. Ait-Ali-Yahia, W. G. Habashi, and M. Robichaud, "Multidomain Spectral Computations of Sound Radiation from Ducted Fans," AIAA Journal, Vol. 37, 1999, pp.296-302.

[12] J. M. Tyler and T. G. Sofrin, "Axial Flow Compressor Noise Studies." SAE Transactions Vol. 70, 1962, pp.308-332.

[13] C. Canuto, M.Y. Hussaini, A. Quarteroni, and T.A. Zang, Spectral Methods in Fluid Dynamics, Springer-Verlag (1988). 
[14] P.L. Roe, "Approximate Riemann Solvers, Parameter Vectors, and Difference Schemes." Journal of Computational Physics Vol. 43, 1981, pp.357-372.

[15] C. Hirsch Numerical Computation of Internal and External Flows. Volume 2: Computational Methods for Inviscid and Viscous Flows, John Wiley \& Sons (1990).
[16] D. Stanescu and W.G. Habashi, "2Nstorage Low Dissipation and Dispersion Runge-Kutta Schemes for Computational Acoustics." Journal of Computational Physics Vol. 143, 1998, pp.674-681.

[17] Balay, S., Gropp, W.D., McInnes, L.C. and Smith, B.F., "PETSc Users Manual", ANL-95/11 Revision 2.1.1, Argonne National Laboratory, 2001.

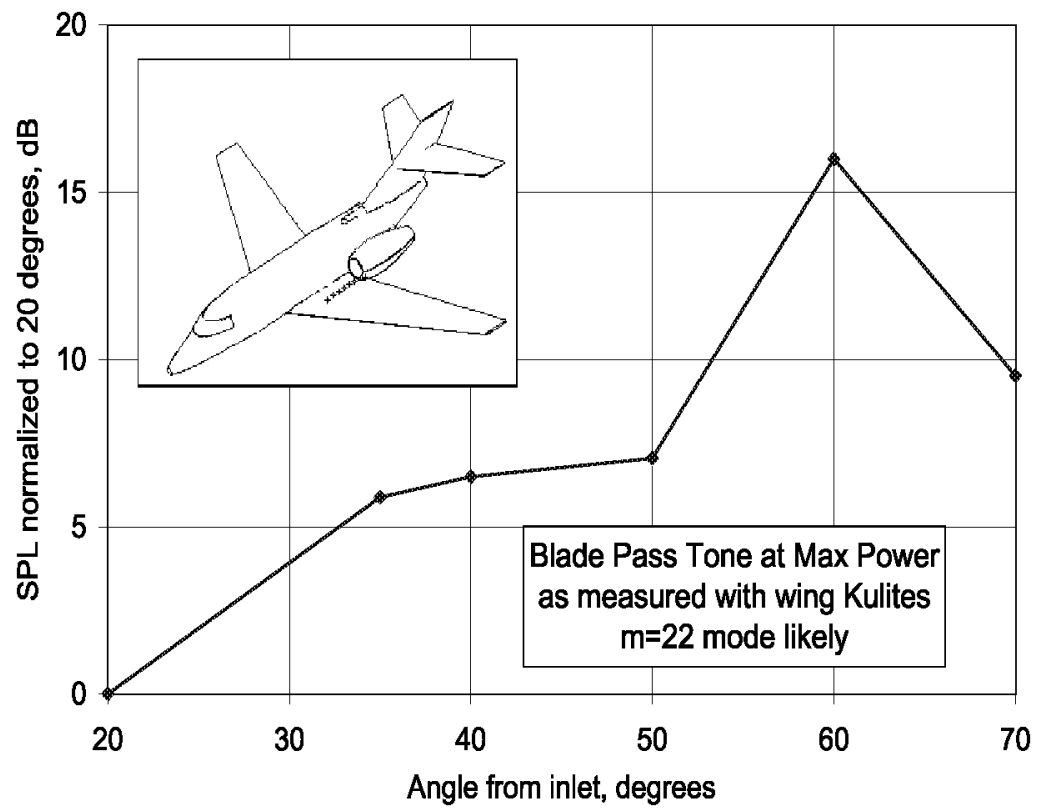

Fig. 1: Location of microphones on the wing and corresponding experimental data.

American Institute of Aeronautics and Astronautics 


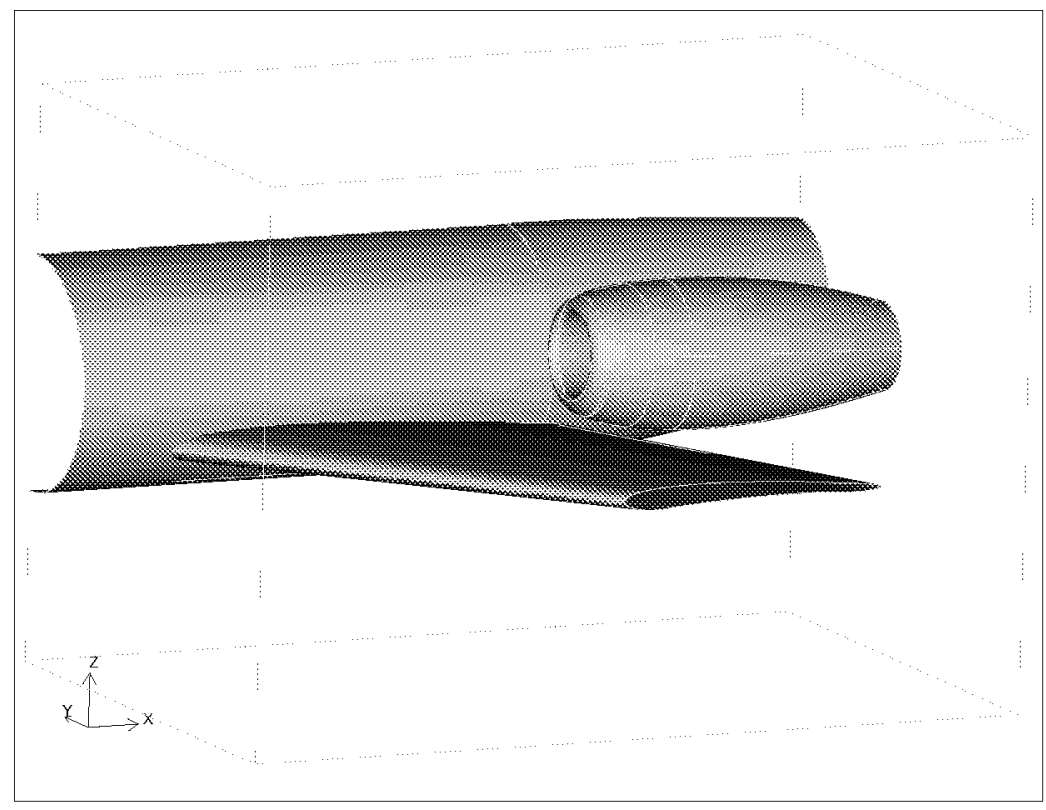

Fig. 2: Computational domain.

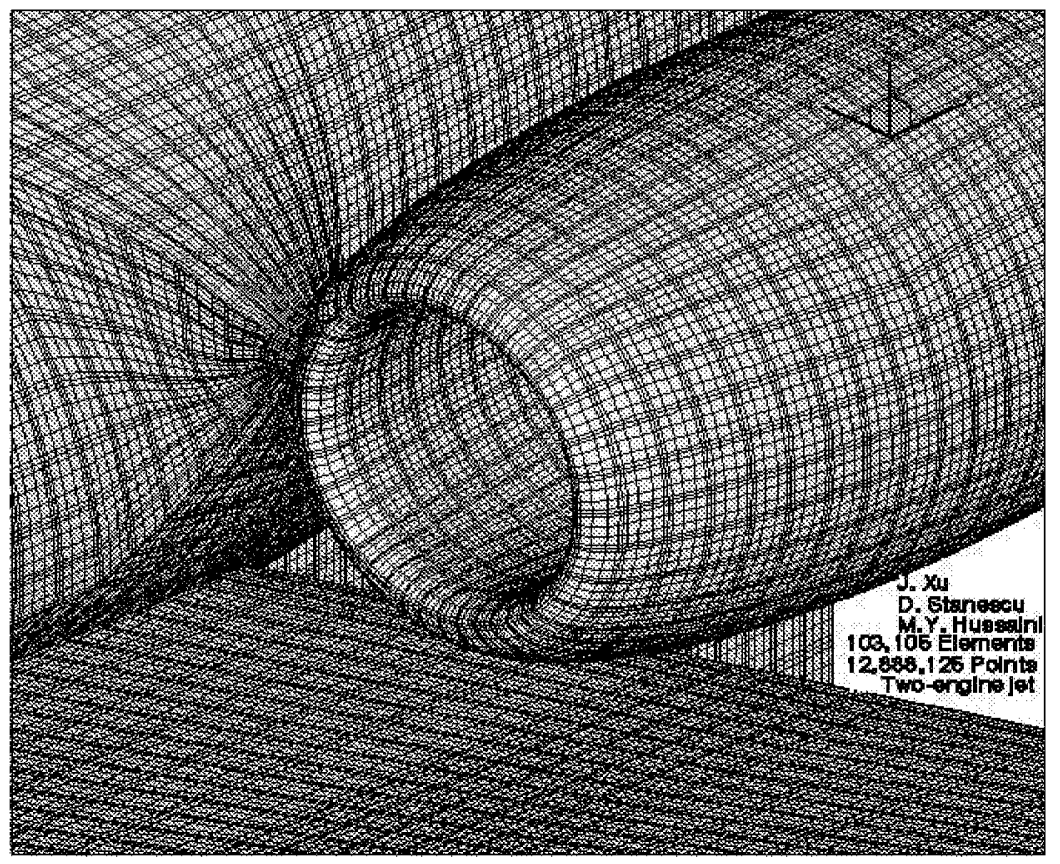

Fig. 3: Mesh on the aircraft surface for $N=5$ (quintic elements).

American Institute of Aeronautics and Astronautics 


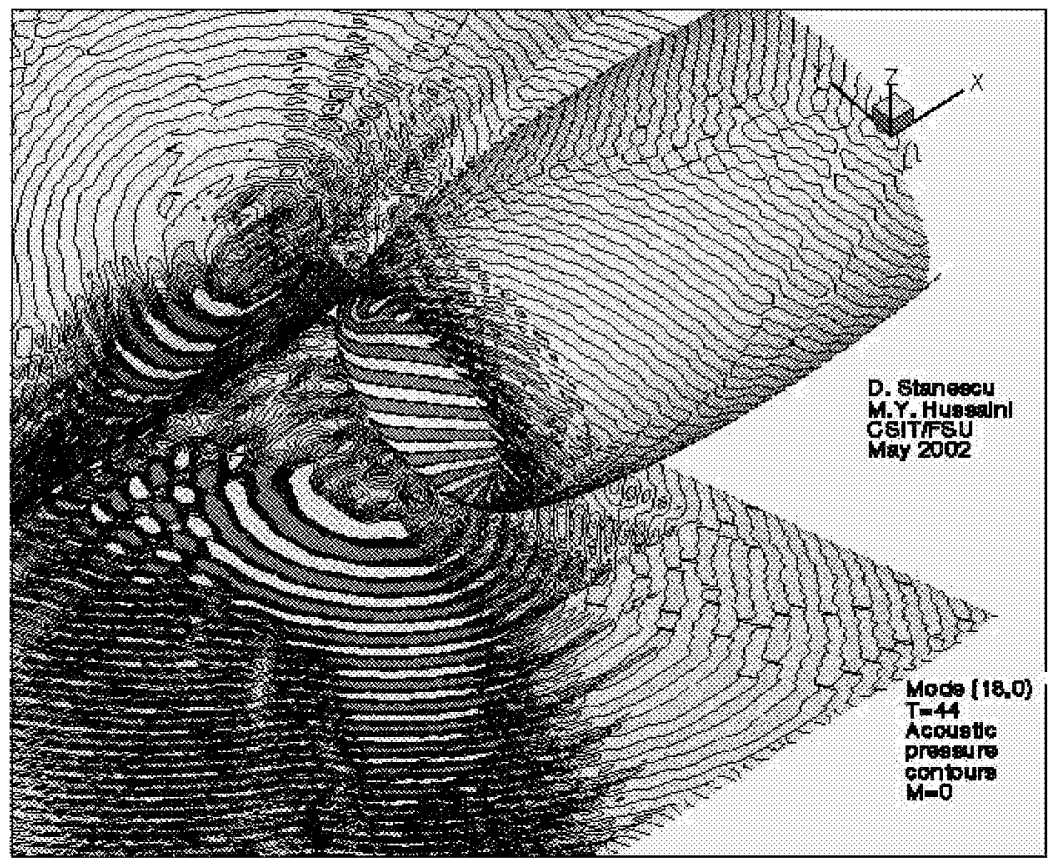

Fig. 4: Acoustic pressure contours on the aircraft surface. Mode $(18,0)$ radiated at $\omega_{r}=26.3$.

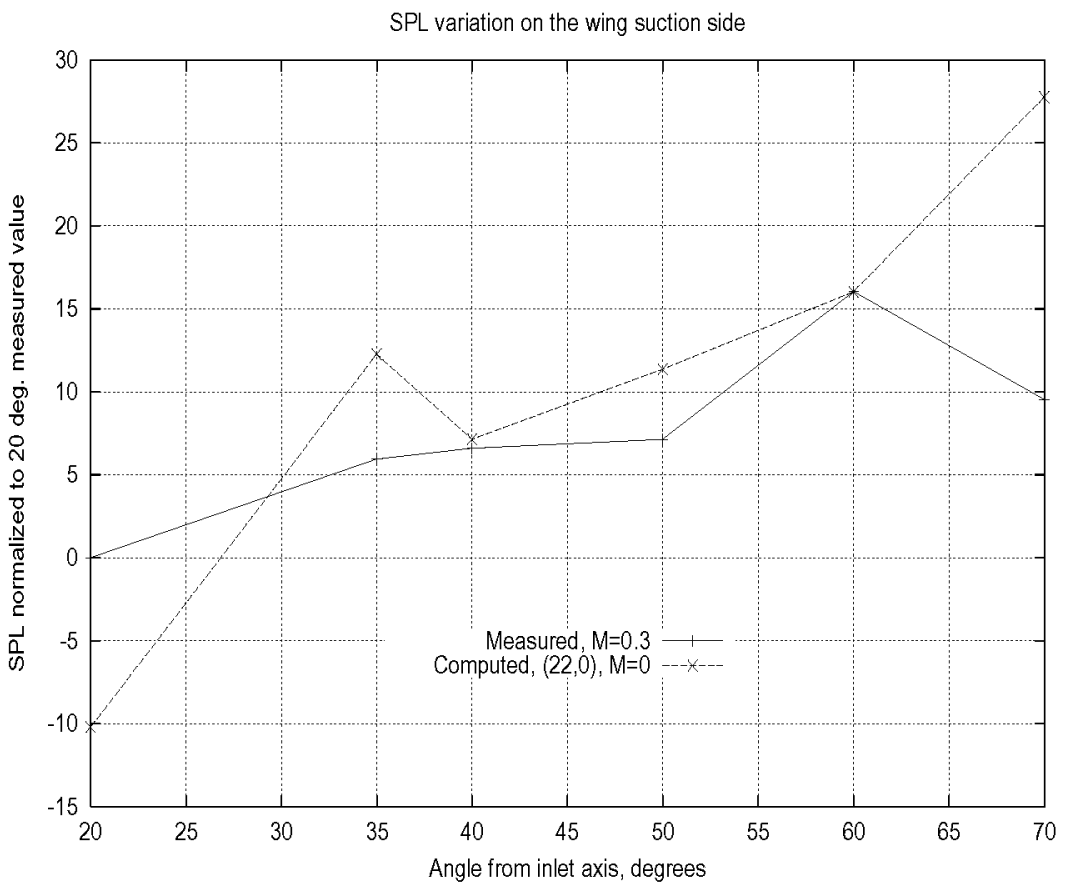

Fig. 5: SPL levels on the wing surface for mode $(22,0)$ radiated at $\omega_{r}=26.3$ in a quiescent medium.

American Institute of Aeronautics and Astronautics 


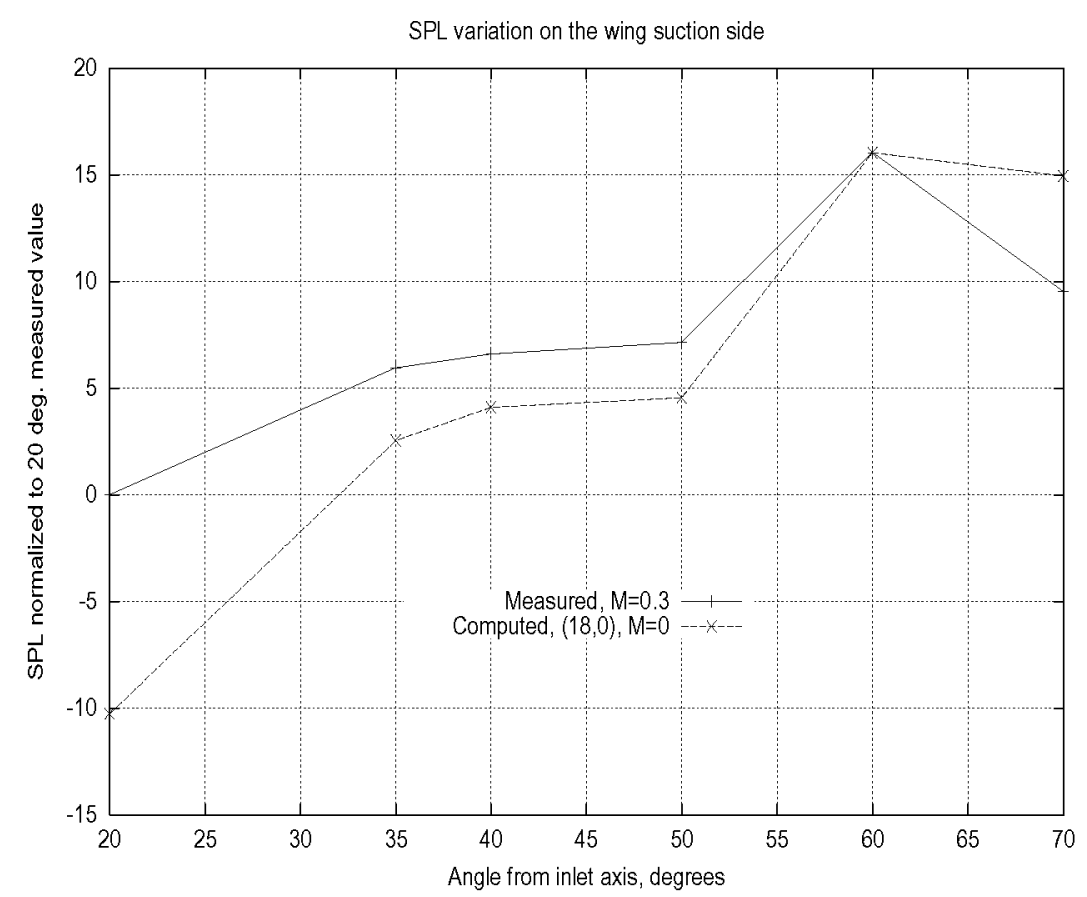

Fig. 6: SPL levels on the wing surface for mode $(18,0)$ radiated at $\omega_{r}=26.3$ in a quiescent medium.

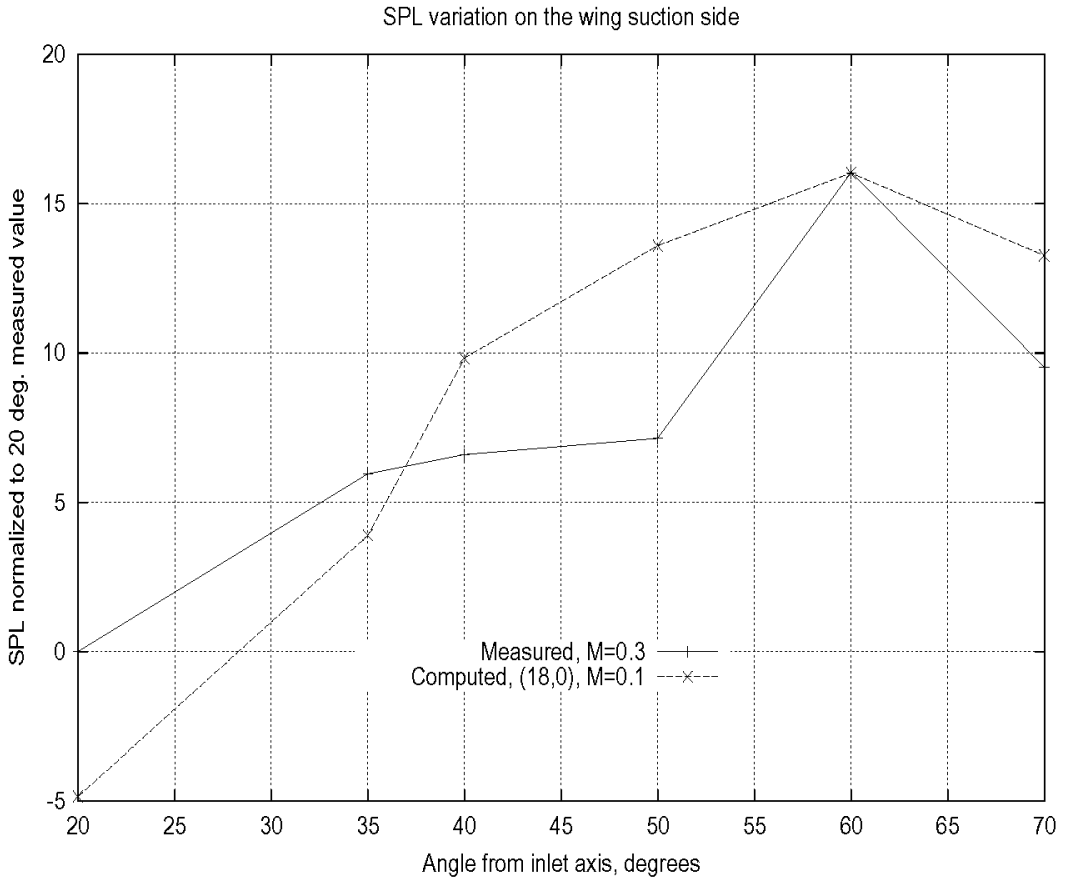

Fig. 7: SPL levels on the wing surface for mode $(18,0)$ radiated at $\omega_{r}=26.3$ in the presence of mean flow.

American Institute of Aeronautics and Astronautics 